\title{
THE HIGHER INTEGRABILITY AND THE VALIDITY OF THE EULER-LAGRANGE EQUATION FOR SOLUTIONS TO VARIATIONAL PROBLEMS*
}

\author{
GIOVANNI BONFANTI ${ }^{\dagger}$, ARRIGO CELLINA $^{\dagger}$, AND MARCO MAZZOLA $^{\dagger}$
}

\begin{abstract}
We prove higher integrability properties of solutions to the problem of minimizing $\int_{\Omega} L(x, u(x), \nabla u(x)) \mathrm{dx}$, where $\xi \mapsto L(x, u, \xi)$ is a convex function satisfying some additional conditions. As an application, we prove the validity of the Euler-Lagrange equation for a class of functionals with growth faster than exponential.
\end{abstract}

Key words. calculus of variations, necessary conditions, Euler-Lagrange equation

AMS subject classification. $49 \mathrm{~K} 10$

DOI. $10.1137 / 110820890$

1. Introduction. In this paper we consider a higher integrability property of a solution $\tilde{u}$ to the problem of minimizing

$$
\int_{\Omega} L(x, u(x), \nabla u(x)) \mathrm{dx} .
$$

More precisely, our aim is to establish the local integrability of the map

$$
\left|\nabla_{\xi} L(\cdot, \tilde{u}(\cdot), \nabla \tilde{u}(\cdot))\right||\nabla \tilde{u}(\cdot)| .
$$

In fact, for Lagrangians $L(x, u, \cdot)$ growing faster than exponential, the integrability of $L(\cdot, \tilde{u}(\cdot), \nabla \tilde{u})$ does not, in general, imply the integrability of $\left|\nabla_{\xi} L(\cdot, \tilde{u}(\cdot), \nabla \tilde{u}(\cdot))\right|$ (see an example in [2]). However, the integrability of (1) is needed both to establish the validity of the Euler-Lagrange equation for the solution to this problem, i.e., in order to prove that the equation

$$
\int_{\Omega}\left[\left\langle\nabla_{\xi} L(x, \tilde{u}(x), \nabla \tilde{u}(x)), \nabla \eta(x)\right\rangle+L_{u}(x, \tilde{u}(x), \nabla \tilde{u}(x)) \eta(x)\right] \mathrm{dx}=0
$$

holds for every admissible variation $\eta$ and to prove additional regularity properties (higher differentiability) of the solution, as in [3].

Clearly, a proof of regularity $\left(\nabla u\right.$ in $\left.L^{\infty}\right)$ of the solution is also a proof of the higher integrability of the solution. In this sense, for the case $L(\xi)=e^{|\xi|^{2}}$, special cases of higher integrability have been obtained by Lieberman [6] and by Naito [7]; Lieberman, in the same paper, considers also a more general Lagrangian but assumes, among other regularity conditions, that the Euler-Lagrange equation admits a $C^{3}$ solution.

In [2], a Lagrangian of the kind $L=e^{f(|\nabla u|)}+g(x, u)$ was considered, where $f$ and $g$ are regular functions satisfying some growth assumptions and $f$ is convex, and a higher integrability result was obtained. The purpose of the present paper

* Received by the editors January 12, 2011; accepted for publication (in revised form) December 19, 2011; published electronically April 17, 2012.

http://www.siam.org/journals/sicon/50-2/82089.html

${ }^{\dagger}$ Dipartimento di Matematica e Applicazioni, Università degli Studi di Milano-Bicocca, Via R. Cozzi 53, I-20125 Milano, Italy (g.bonfanti3@campus.unimib.it, arrigo.cellina@unimib.it, m.mazzola7@campus.unimib.it). 
is twofold: first we wish to present a more general result, suited for being used in the investigation of further regularity properties of the solution; second, we wish to use the higher integrability property to establish the validity of the Euler-Lagrange equation for a class of Lagrangians growing faster than exponential.

It is well known, in fact (see, e.g., [4]), that so far, the validity of the EulerLagrange equation for Lagrangians of general form has been established only for Lagrangians growing at most exponentially; Lieberman and Naito consider the case $L=e^{|\nabla u|^{2}}$; more recently, Degiovanni and Marzocchi in [5] consider functionals of the form $\int L(\nabla u(x)) \mathrm{dx}+\varphi(\mathrm{u})$, where $\varphi \in W^{-1, p^{\prime}}$, without any upper growth condition on $L$, and in [1], $\varphi(u)$ is replaced by a more general term $g(x, u)$ concave w.r.t. $u$. However, the few results proved so far for integrands having growth faster than exponential hold only for Lagrangians of a very special form.

The proof of the higher integrability result, which will be presented below, is independent on the validity of the Euler-Lagrange equation; this fact prompted us to try to use the higher integrability property to extend the validity of the EulerLagrange equation beyond exponential growth. A result along these lines is presented in the second part of the paper: in it, we allow the growth of $L$ with respect to $\xi$ to be approximately up to $|\xi|^{|\xi|} \equiv \exp (|\xi| \log |\xi|)$.

2. Assumptions and higher integrability results. Some results in this paper will depend on the properties of the polar or Legendre-Fenchel transform $L^{*}$ of a convex function $L$, defined by

$$
L^{*}(p)=\sup \{\langle p, \xi\rangle-L(\xi)\}
$$

for its properties, we refer to [8].

We shall consider Lagrangians $L$ satisfying the following convexity and regularity assumptions.

Assumption A. $L(x, u, \xi)$ is nonnegative and positive whenever $\xi \neq 0$, and the map $t \mapsto L(x, u, t \xi)$ is nondecreasing for $t \geq 0$. In addition, for every $(x, u)$, the restriction to the set $|\xi| \geq 1$ of the mapping $\xi \mapsto L(x, u, \xi)$ is the restriction to the same set of a convex function. Moreover, $L(x, u, \xi)$ is $C^{1}(u \times \xi)$ for each fixed $x$ and measurable in $x$ for each fixed $(u, \xi)$, and it is such that for every $\omega \subset \subset \Omega$ and $U$ there exist constants $M=M(\omega, U), K=K(\omega, U)$, and, for every $R$, a function $\alpha_{\omega, U, R}$ in $L^{1}(\omega)$ such that for almost every $x \in \omega$ and for every $|u| \leq U$ we have

(i) for every $\xi \in R^{n},\left|\frac{\partial L(x, u, \xi)}{\partial u}\right| \leq K L(x, u, \xi)$;

(ii) $\sup \left\{\left|\nabla_{\xi} L(x, u, \xi)\right|:|u| \leq U ;|\xi| \leq R\right\} \leq \alpha_{\omega, U, R}(x)$;

(iii) $\left\langle\nabla_{\xi} L(x, u, \xi), \xi\right\rangle \geq M\left|\nabla_{\xi} L(x, u, \xi)\right||\xi|$.

The higher integrability results will depend on the validity of the following condition. In it, and for the remainder of the paper, for an open $O \subset \subset \Omega$ and $\delta>0$, we set $O_{\delta}=O+B(0, \delta)$. Explicit classes of Lagrangians satisfying Condition $\mathrm{C}$ will be provided by Theorem 2 .

Condition C. For every open $O \subset \subset \Omega, \delta^{0}>0$, and $U$ there exist a constant $\delta \leq \delta^{0}$ such that $\bar{O}_{\delta}$ is in $\Omega$, a Lipschitzian function $\eta \in C_{c}\left(O_{\delta}\right)$ with $\eta(x) \geq 0$ and $\eta(x)=1$ on $O$, and constants $\tilde{K}=\tilde{K}\left(U, O_{\delta}\right) \geq 0$ and $\tilde{R}=\tilde{R}\left(U, O_{\delta}\right)$ such that for every $\xi$ with $|\xi| \geq \tilde{R}$, for every $u$ with $|u| \leq U$, for almost every $x \in O_{\delta}$, and for every $\varepsilon>0$ sufficiently small, we have

$$
\log L(x, u-\varepsilon \eta u, \xi(1-\varepsilon \eta)-\varepsilon u \nabla \eta)-\log L(x, u, \xi) \leq \varepsilon \tilde{K} .
$$

The next theorem infers the higher integrability result from the validity of Condition $\mathrm{C}$. 
Theorem 1. Let L satisfy Assumption A and Condition C. Let $\tilde{u}$ be a locally bounded solution to the problem of minimizing

$$
\int_{\Omega} L(x, u(x), \nabla u(x)) \mathrm{d} x
$$

on $u^{0}+W_{0}^{1,1}(\Omega)$. Then,

$$
\left|\nabla_{\xi} L(\cdot, \tilde{u}(\cdot), \nabla \tilde{u}(\cdot))\right||\nabla \tilde{u}(\cdot)| \in \mathrm{L}_{l o c}^{1}(\Omega) .
$$

Proof. (a) Fix $O \subset \subset \Omega$. It is enough to prove the existence of $H_{1}$ such that

$$
\int_{O}\left\langle\nabla_{\xi} L(x, \tilde{u}(x), \nabla \tilde{u}(x)), \nabla \tilde{u}(x)\right\rangle \mathrm{d} x \leq H_{1} .
$$

In fact, if this is true, taking $O$ to be $\omega$ in Assumption A, point (iii) proves the claim.

Hence, let $O_{\delta^{0}} \subset \subset \Omega$, and let $U$ be a bound for $|\tilde{u}|$ on $O_{\delta^{0}}$. Let $\delta, \eta$ and the constants $\tilde{R}$ and $\tilde{K}$ be provided by Condition $\mathrm{C}$ (we assume $\tilde{R} \geq 1$ ).

Since $\tilde{u}$ is a solution, for the variation $-\varepsilon \eta \tilde{u}$, with $\varepsilon>0$, we obtain

$$
0 \leq \frac{1}{\varepsilon} \int_{O_{\delta}}[L(x, \tilde{u}-\varepsilon \eta \tilde{u}, \nabla \tilde{u}(1-\varepsilon \eta)-\varepsilon \tilde{u} \nabla \eta)-L(x, \tilde{u}, \nabla \tilde{u})] \mathrm{d} x .
$$

We have

$$
\begin{aligned}
L(x, \tilde{u} & -\varepsilon \eta \tilde{u}, \nabla \tilde{u}(1-\varepsilon \eta)-\varepsilon \tilde{u} \nabla \eta)-L(x, \tilde{u}, \nabla \tilde{u}) \\
& =\varepsilon \int_{0}^{1}\left[\frac{\partial L}{\partial u}(-\eta \tilde{u})+\left\langle\nabla_{\xi} L,-\eta \nabla \tilde{u}-\tilde{u} \nabla \eta\right\rangle\right] \mathrm{d} s,
\end{aligned}
$$

where $\frac{\partial L}{\partial u}$ and $\nabla_{\xi} L$ are computed at $(x, \tilde{u}-s \varepsilon \eta \tilde{u}, \nabla \tilde{u}(1-s \varepsilon \eta)-s \varepsilon \tilde{u} \nabla \eta)$; hence, as $\varepsilon \rightarrow 0$, by the continuity of the partial derivatives of $L$,

$$
\begin{aligned}
& \frac{L(x, \tilde{u}-\varepsilon \eta \tilde{u}, \nabla \tilde{u}(1-\varepsilon \eta)-\varepsilon \tilde{u} \nabla \eta)-L(x, u, \nabla \tilde{u})}{\varepsilon} \\
& \rightarrow \frac{\partial L}{\partial u}(-\eta \tilde{u})+\left\langle\nabla_{\xi} L,-\eta \nabla \tilde{u}-\tilde{u} \nabla \eta\right\rangle,
\end{aligned}
$$

pointwise in $x$, and with the right-hand side computed at $(x, \tilde{u}(x), \nabla \tilde{u}(x))$. Set $O_{\delta}{ }^{-}=$ $\left\{x \in O_{\delta}:|\nabla \tilde{u}(x)|<\tilde{R}\right\}$ and $O_{\delta}{ }^{+}=\left\{x \in O_{\delta}:|\nabla \tilde{u}(x)| \geq \tilde{R}\right\}:$ on $O_{\delta}{ }^{-}$, the left-hand side of (4) is uniformly bounded so that for every $\varepsilon$ and for some $\tilde{M}$ we have

$$
\left|\frac{1}{\varepsilon} \int_{O_{\delta}^{-}}[L(x, \tilde{u}-\varepsilon \eta \tilde{u}, \nabla \tilde{u}(1-\varepsilon \eta)-\varepsilon \tilde{u} \nabla \eta)-L(x, \tilde{u}(x), \nabla \tilde{u}(x))] \mathrm{d} x\right| \leq \tilde{M} .
$$

(b) On $O_{\delta}{ }^{+}$, consider the constant $\tilde{K}$ : setting

$$
\tilde{\ell}_{\varepsilon}(x)=\log L(x, \tilde{u}(x)-\varepsilon \eta(x) \tilde{u}(x), \nabla \tilde{u}(x)(1-\varepsilon \eta(x))-\varepsilon \tilde{u}(x) \nabla \eta(x)),
$$

from (3) we have

$$
\begin{aligned}
-\tilde{M} & \leq \int_{O_{\delta}+}\left(\frac{e^{\tilde{\ell}_{\varepsilon}}-e^{\log L(x, \tilde{u}(x), \nabla \tilde{u}(x))}}{\varepsilon}\right) \mathrm{d} x \\
& =\int_{O_{\delta}+}\left(\frac{e^{\tilde{\ell}_{\varepsilon}-\varepsilon \tilde{K}+\varepsilon \tilde{K}}-e^{\log L(x, \tilde{u}(x), \nabla \tilde{u}(x))}}{\varepsilon}\right) \mathrm{d} x \\
& =\int_{O_{\delta}+} e^{\tilde{\ell}_{\varepsilon}-\varepsilon \tilde{K}}\left[\frac{e^{\varepsilon \tilde{K}}-1+1-e^{\log L(x, \tilde{u}(x), \nabla \tilde{u}(x))-\tilde{\ell}_{\varepsilon}+\varepsilon \tilde{K}}}{\varepsilon}\right] \mathrm{d} x,
\end{aligned}
$$


i.e.,

$$
\begin{aligned}
& \tilde{M}+\int_{O_{\delta}+} e^{\tilde{\ell}_{\varepsilon}-\varepsilon \tilde{K}}\left[\frac{e^{\varepsilon \tilde{K}}-1}{\varepsilon}\right] \mathrm{d} x \\
& \quad \geq \int_{O_{\delta}^{+}} e^{\tilde{\ell}_{\varepsilon}-\varepsilon \tilde{K}}\left[\frac{e^{\log L(x, \tilde{u}(x), \nabla \tilde{u}(x))-\tilde{\ell}_{\varepsilon}+\varepsilon \tilde{K}}-1}{\varepsilon}\right] \mathrm{d} x .
\end{aligned}
$$

Since on $O_{\delta}{ }^{+}, \tilde{\ell}_{\varepsilon}(x)-\varepsilon \tilde{K} \leq \log L(x, \tilde{u}(x), \nabla \tilde{u}(x))$ and also $\frac{e^{\varepsilon \tilde{K}}-1}{\varepsilon} \leq \tilde{K} e^{\tilde{K}}$, the lefthand side of (5) is bounded by

$$
\tilde{M}+\tilde{K} e^{\tilde{K}} \int_{\Omega} L(x, \tilde{u}(x), \nabla \tilde{u}(x)) \mathrm{d} x=H,
$$

independent of $\varepsilon$. (c) Consider the right-hand side. For fixed $x$ we have

$$
\log L(x, \tilde{u}(x), \nabla \tilde{u}(x))-\tilde{\ell}_{\varepsilon}(x)=-\varepsilon\left[\frac{1}{L} \frac{\partial L}{\partial u}(-\eta \tilde{u})+\frac{1}{L}\left\langle\nabla_{\xi} L,-\eta \nabla \tilde{u}-\tilde{u} \nabla \eta\right\rangle\right]+o(\varepsilon)
$$

so that, as $\varepsilon \rightarrow 0$, pointwise w.r.t. $x$,

$$
\frac{e^{\log L(x, \tilde{u}, \nabla \tilde{u})-\tilde{\ell}_{\varepsilon}+\varepsilon \tilde{K}}-1}{\varepsilon} \rightarrow \tilde{K}+\frac{1}{L} \frac{\partial L}{\partial u} \eta \tilde{u}+\frac{1}{L}\left\langle\nabla_{\xi} L, \eta \nabla \tilde{u}+\tilde{u} \nabla \eta\right\rangle .
$$

In addition, by $(2), \log L(x, \tilde{u}(x), \nabla \tilde{u}(x))-\tilde{\ell}_{\varepsilon}(x)+\varepsilon \tilde{K} \geq 0$ so that the left-hand side of (6) is nonnegative and so is its limit, $\tilde{K}+\frac{1}{L} \frac{\partial L}{\partial u} \eta \tilde{u}+\frac{1}{L}\left\langle\nabla_{\xi} L, \eta \nabla \tilde{u}+\tilde{u} \nabla \eta\right\rangle$. Finally, pointwise, $e^{\tilde{\ell}_{\varepsilon}-\varepsilon \tilde{K}} \rightarrow e^{\log L(x, \tilde{u}, \nabla \tilde{u})}$. Hence, applying Fatou's lemma, we obtain

$$
\int_{O_{\delta}^{+}} L(x, \tilde{u}, \nabla \tilde{u})\left[\tilde{K}+\frac{\frac{\partial L}{\partial u}}{L} \eta \tilde{u}+\frac{1}{L}\left\langle\nabla_{\xi} L, \eta \nabla \tilde{u}+\tilde{u} \nabla \eta\right\rangle\right] \leq H,
$$

i.e.,

$$
\int_{O_{\delta}^{+}}\left[\tilde{K} L(x, \tilde{u}, \nabla \tilde{u})+\frac{\partial L}{\partial u} \eta \tilde{u}+\left\langle\nabla_{\xi} L, \eta \nabla \tilde{u}+\tilde{u} \nabla \eta\right\rangle\right] \leq H .
$$

Since the integrand above is nonnegative, we have obtained, in particular, that

$$
\int_{O \cap O_{\delta}^{+}}\left[\tilde{K} L(x, \tilde{u}, \nabla \tilde{u})+\frac{\partial L}{\partial u} \eta \tilde{u}+\left\langle\nabla_{\xi} L, \eta \nabla \tilde{u}+\tilde{u} \nabla \eta\right\rangle\right] \leq H .
$$

On $O$ we have that $\eta \equiv 1, \tilde{u}$ is bounded, and that, by (i) of Assumption A, there exists $K$ such that

$$
\left|\frac{\partial L(x, \tilde{u}(x), \nabla \tilde{u}(x))}{\partial u}\right| \leq K L(x, \tilde{u}(x), \nabla \tilde{u}(x))
$$

hence there exists $H^{+}$such that

$$
\int_{O \cap O_{\delta}^{+}}\left\langle\nabla_{\xi} L(x, \tilde{u}(x), \nabla \tilde{u}(x)), \nabla \tilde{u}(x)\right\rangle \mathrm{d} x \leq H^{+} .
$$


Consider $O \cap O_{\delta}^{-}$; by Assumption A(ii), we have that

$$
\left|\left\langle\nabla_{\xi} L(x, \tilde{u}(x), \nabla \tilde{u}(x)), \nabla \tilde{u}(x)\right\rangle\right| \leq \tilde{R} \cdot \alpha_{\omega, U, \tilde{R}}(x)
$$

on $O \cap O_{\delta}^{-}$. Hence we have obtained that the integral

$$
\int_{O}\left\langle\nabla_{\xi} L(x, \tilde{u}(x), \nabla \tilde{u}(x)), \nabla \tilde{u}(x)\right\rangle \mathrm{d} x
$$

is bounded, thus proving the theorem.

It is easy to show that the Lagrangians of exponential growth satisfy Condition C. However, the following result shows that this condition is satisfied by a substantially larger class of functions. We shall need the following.

Definition 1. A convex function $\Lambda \in C^{1}(\Re), \Lambda(t)>0$ for $t \neq 0$, is called a comparison function for $L$ if for every $U$ there exist constants $K_{0}, K_{1}$, and $K_{2}$ such that for almost every $x \in \omega,|u| \leq U$ and $|\xi| \geq 1$ imply

(i) $\Lambda(|\xi|) \leq K_{0} L(x, u, \xi)$;

(ii) $K_{1} \Lambda^{\prime}(|\xi|) \leq\left|\nabla_{\xi} L(x, u, \xi)\right| \leq K_{2} \Lambda^{\prime}(|\xi|)$.

We shall also refer to the following.

Exponential GROWTH CONDITION. For every open $O \subset \subset \Omega$ and $U$ there exists a constant $c$ such that for almost every $x \in O,|u| \leq U$ and $|\xi| \geq 1$ imply $\left|\nabla_{\xi} L(x, u, \xi)\right| \leq c \cdot L(x, u, \xi)$.

THEOREM 2. Let L satisfy Assumption A. Assume that either

(i) L satisfies the exponential growth condition or

(ii) for $|\xi| \geq 1$, the map $\log (L(x, u, \cdot))$ is the restriction to the set $|\xi| \geq 1$ of a convex function; there exists a comparison function $\Lambda$ such that $\mathcal{L}(\cdot)=\log (\Lambda(\cdot))$ is convex and such that $\mathcal{L}^{*}$ is defined on $\Re$. Assume that

$$
\int^{\infty} \frac{1}{z \partial\left(\mathcal{L}^{*}\right)(z)} \mathrm{d} z<\infty
$$

Then Condition $\mathrm{C}$ is satisfied.

Remarks. For every sufficiently large $z, 0 \notin \partial\left(\mathcal{L}^{*}\right)(z)$; if this was not the case, in fact, the map $z \mapsto\left(\mathcal{L}^{*}\right)(z)$ would be constant and $\mathcal{L}$, hence $\Lambda$, would be defined on a single point.

The map $t \mapsto \exp \left(|t|^{p}\right)$ for $p>1$ satisfies condition (ii) but not condition (i); the map $t \mapsto \exp (\exp (t))$ satisfies neither condition (i) nor condition (ii). in $[2])$.

In the proof of Theorem 2, we shall need the following preliminary result (Lemma 1

Lemma 1. Let $G: \Re \rightarrow 2^{\Re}$ be upper semicontinuous, strictly increasing, and such that $G(0)=\{0\}$. Assume that for a selection $g$ from $G$

$$
\int^{\infty} g(1 / s) \mathrm{d} s<\infty
$$

Then the implicit Cauchy problem

$$
\left\{\begin{array}{l}
x(t) \in G\left(x^{\prime}(t)\right) \\
x(0)=0
\end{array}\right.
$$

admits a solution $\tilde{x}$, positive on some interval $(0, \tau]$. 
Proof of Theorem 2. Fix $O, \delta^{0}$, and $U$. Let $0<\delta \leq \delta^{0}$ be such that $\bar{O}_{\delta}$ is in $\Omega$. For a fixed variation $\eta$, we shall use the notation

$$
\ell_{\varepsilon}(x, u, \xi)=\log L(x, u-\varepsilon \eta u, \xi(1-\varepsilon \eta)-\varepsilon u \nabla \eta) .
$$

(a) In case (i), choose any Lipschitz continuous function $\eta \in C_{c}\left(O_{\delta}\right)$ with $\eta(x) \geq 0$ and $\eta(x)=1$ on $O$, and set $\mu=\sup |\nabla \eta|$. Choose $\tilde{R}=\max \{4,2 U \mu\}$ so that for $\varepsilon \leq \frac{1}{2}$, $|\xi| \geq \tilde{R}$ implies, for $s \in[0,1],|\xi(1-\varepsilon \eta)-s \varepsilon u \nabla \eta| \geq 1$.

Fix $u,|u| \leq U$ and notice that for $s \in[0,1]$ we have $|u-s \varepsilon \eta u| \leq U$. We have

$$
\begin{aligned}
\ell_{\varepsilon}(x, u, \xi)-\log L(x, u, \xi)= & \int_{0}^{1} \frac{\partial \log L(x, u-s \varepsilon \eta u, \xi(1-\varepsilon \eta)-\varepsilon u \nabla \eta)}{\partial u}(-\varepsilon \eta u) \mathrm{d} s \\
& +\int_{0}^{1}\left\langle\nabla_{\xi} \log L(x, u, \xi(1-\varepsilon \eta)-s \varepsilon u \nabla \eta),-\varepsilon u \nabla \eta\right\rangle \mathrm{d} s \\
& +\log L(x, u, \xi(1-\varepsilon \eta))-\log L(x, u, \xi) .
\end{aligned}
$$

By Assumption A, $t \mapsto L(x, u, t \xi)$ is nondecreasing with respect to $t$ on $\{t \geq 0\}$; hence the third term on the right-hand side is nonpositive. Moreover, $\left|\frac{\partial \log \bar{L}}{\partial u}\right|=$ $\frac{1}{L}\left|\frac{\partial L}{\partial u}\right| \leq K$ so that the first term is bounded by $\varepsilon$ times a constant. By the exponential growth assumption, $\left|\nabla_{\xi} \log L\right|=\frac{\left|\nabla_{\xi} L\right|}{L} \leq c$, and hence the same is true for the second term.

(b) Consider case (ii). From

$$
\ell_{\varepsilon}(x, u, \xi)-\log L(x, u, \xi)=\varepsilon(-\eta u) \int_{0}^{1} \frac{1}{L} \frac{\partial L}{\partial u} \mathrm{~d} s+\varepsilon \int_{0}^{1}\left\langle\frac{\nabla_{\xi} L}{L},-\eta \xi-u \nabla \eta\right\rangle \mathrm{d} s,
$$

where the first integrand is evaluated at $(x, u-s \varepsilon \eta u, \xi(1-\varepsilon \eta)-\varepsilon u \nabla \eta)$ and the second at $(x, u, \xi(1-s \varepsilon \eta)-s \varepsilon u \nabla \eta)$, we obtain

$$
\ell_{\varepsilon}(x, u, \xi)-\log L(x, u, \xi) \leq \varepsilon\left[K U+\left\langle\frac{\nabla_{\xi} L}{L}\left(x, u, \xi_{\varepsilon}\right),-\eta \xi-u \nabla \eta\right\rangle\right],
$$

where $\xi_{\varepsilon}=\left(1-s_{\varepsilon} \varepsilon \eta\right) \xi-s_{\varepsilon} \varepsilon u \nabla \eta$ for some $0 \leq s_{\varepsilon} \leq 1$.

For $z \neq 0$, set

$$
G(1 / z)=\frac{1}{z} \frac{7 U}{M \partial\left(\mathcal{L}^{*}\right)(z)} .
$$

From the assumption of convexity, $\partial\left(\mathcal{L}^{*}\right)$ is nonincreasing as a function of $\frac{1}{z}$ and $\frac{7 U}{M \partial\left(\mathcal{L}^{*}\right)(z)}$ is nondecreasing as a function of $\frac{1}{z}$ so that $G$ satisfies the assumptions of Lemma 1.

Consider $\tilde{x}$, the solution to $\tilde{x} \in G\left(\tilde{x}^{\prime}\right)$, provided by Lemma 1 , defined and positive on $(0, \tau]$. Possibly decreasing $\tau$, we can assume, without loss of generality, that

$$
x^{\prime}(t) \leq 1 \text { for all } t \in(0, \tau] .
$$

Notice that from the inclusion $x(t) \in G\left(x^{\prime}(t)\right)$ we infer that $x^{\prime}>0$ on $(0, \tau]$, hence that $x$ is strictly increasing, so that $x^{\prime}$ is strictly increasing as well. Set $\delta_{\tau}=\min \{\tau, \delta\}$ and define $\eta$ as follows: let $d(x)$ be the distance from a point $x \in O_{\delta_{\tau}}$ to $\partial O_{\delta_{\tau}}$ and set

$$
\eta(x)=\inf \left\{\frac{1}{\tilde{x}\left(\delta_{\tau}\right)} \tilde{x}(d(x)), 1\right\}
$$


so that, in particular, $\eta=1$ on $O$. Almost everywhere, $d$ is differentiable with $|\nabla d|=1$ and, at a point of differentiability, we have

$$
\nabla \eta(x)= \begin{cases}0 & \text { if } d(x)>\delta_{\tau} \\ \frac{1}{\tilde{x}\left(\delta_{\tau}\right)} \tilde{x}^{\prime}(d(x)) \nabla d(x) & \text { if } d(x)<\delta_{\tau}\end{cases}
$$

Hence, a.e., we have that $|\nabla \eta| \leq \frac{1}{\tilde{x}\left(\delta_{\tau}\right)} \tilde{x}^{\prime}\left(\delta_{\tau}\right)$ and that either $\nabla \eta=0$ or

$$
\begin{aligned}
\eta(x) & =\frac{1}{\tilde{x}\left(\delta_{\tau}\right)} \tilde{x}(d(x))=\frac{1}{\tilde{x}\left(\delta_{\tau}\right)} \tilde{x}^{\prime}(d(x)) \frac{7 U}{M \partial\left(\mathcal{L}^{*}\right)\left(\frac{1}{\tilde{x}^{\prime}(d(x))}\right)} \\
& =h\left(\tilde{x}\left(\delta_{\tau}\right)|\nabla \eta(x)|\right)|\nabla \eta(x)|
\end{aligned}
$$

where we have set

$$
h(z)=\frac{7 U}{M \partial\left(\mathcal{L}^{*}\right)\left(\frac{1}{z}\right)},
$$

an increasing function.

Consider the term $\varepsilon\left\langle\frac{\nabla \xi L}{L}\left(x, u, \xi_{\varepsilon}\right),-\eta \xi-u \nabla \eta\right\rangle$ in (10). Set $\bar{\xi}=(1-\varepsilon \eta) \xi-\varepsilon u \nabla \eta$.

Set $\mu_{1}=\sup |\nabla \eta|$ and $\tilde{R}=2+U \mu_{1}$ so that for $\varepsilon \leq \frac{1}{2},|\xi| \geq \tilde{R}$ implies that both $\left|\xi_{\varepsilon}\right| \geq 1$ and $|\bar{\xi}| \geq 1$.

For those $x$ such that

$$
\left\langle\frac{\nabla_{\xi} L}{L}\left(x, u, \xi_{\varepsilon}\right),-\eta \xi-u \nabla \eta\right\rangle \leq 0,
$$

any $\tilde{K} \geq K U$ will do to prove the result. Moreover, by Assumption A, we have $\frac{d}{d s} L(x, u, \xi(1-s \varepsilon \eta)) \leq 0$, i.e., $\left\langle\nabla_{\xi} L(x, u, \xi(1-s \varepsilon \eta)),-\eta \xi\right\rangle \leq 0$, so that

$$
\left\langle\frac{\nabla_{\xi} L}{L}(x, u, \xi(1-s \varepsilon \eta)),-\eta \xi\right\rangle \leq 0
$$

from this we infer that, when $\nabla \eta(x)=0,(14)$ holds.

Hence, we are left to consider those $x$ such that, at once,

$$
\left\langle\frac{\nabla_{\xi} L}{L}\left(x, u, \xi_{\varepsilon}\right),-\eta \xi-u \nabla \eta\right\rangle>0
$$

and $\eta(x)=|\nabla \eta(x)| h\left(\tilde{x}\left(\delta_{\tau}\right)|\nabla \eta(x)|\right)$.

Given any $v, w \in \Re^{n}$, from the assumption of convexity of $\log L(x, u, \cdot)$, we obtain that its gradient is monotonic, i.e., that

$$
\left(s_{1}-s_{2}\right)\left\langle\frac{\nabla_{\xi} L}{L}\left(x, u, v+s_{1} w\right)-\frac{\nabla_{\xi} L}{L}\left(x, u, v+s_{2} w\right), w\right\rangle \geq 0,
$$

i.e., that the mapping $s \mapsto\left\langle\frac{\nabla_{\xi} L}{L}(x, u, v+s w), w\right\rangle$ is nondecreasing. Hence, from the inequality

$$
\left\langle\frac{\nabla_{\xi} L}{L}\left(x, u, \xi_{\varepsilon}\right),-\eta \xi-u \nabla \eta\right\rangle>0
$$

we obtain $\left\langle\frac{\nabla_{\xi} L}{L}(x, u, \bar{\xi}),-\eta \xi-u \nabla \eta\right\rangle>0$. We infer that

$$
\left\langle\frac{\nabla_{\xi} L}{L}(x, u, \bar{\xi}), \xi\right\rangle<\left\langle\frac{\nabla_{\xi} L}{L}(x, u, \bar{\xi}),-u \frac{\nabla \eta}{\eta}\right\rangle \leq\left|\frac{\nabla_{\xi} L}{L}(x, u, \bar{\xi})\right| U \frac{|\nabla \eta|}{\eta} .
$$


Recalling Assumption A(iii), we have

$$
\begin{aligned}
\left\langle\frac{\nabla_{\xi} L}{L}(x, u, \bar{\xi}), \xi\right\rangle & =\left\langle\frac{\nabla_{\xi} L}{L}(x, u, \bar{\xi}), \bar{\xi}\right\rangle-\varepsilon\left\langle\frac{\nabla_{\xi} L}{L}(x, u, \bar{\xi}),-\eta \xi-u \nabla \eta\right\rangle \\
& \geq\left|\frac{\nabla_{\xi} L}{L}(x, u, \bar{\xi})\right|[M|\bar{\xi}|-\varepsilon \eta|\xi|-\varepsilon U|\nabla \eta|] .
\end{aligned}
$$

From inequalities (15) and (16), we infer

$$
U \frac{|\nabla \eta|}{\eta}>M|\bar{\xi}|-\varepsilon \eta|\xi|-\varepsilon U|\nabla \eta| \geq M[(1-\varepsilon \eta)|\xi|-\varepsilon U|\nabla \eta|]-\varepsilon \eta|\xi|-\varepsilon U|\nabla \eta|,
$$

i.e.,

$$
U \frac{|\nabla \eta|}{\eta}+\varepsilon|\nabla \eta| U[M+1]>|\xi|[M(1-\varepsilon \eta)-\varepsilon \eta] .
$$

We are free to assume $M<1$; taking $\varepsilon<\frac{M}{4}$, we finally have

$$
3 U \frac{|\nabla \eta|}{\eta}>U \frac{|\nabla \eta|}{\eta}+\varepsilon|\nabla \eta| U[M+1]>|\xi|[M(1-\varepsilon \eta)-\varepsilon \eta]>\frac{1}{2} M|\xi|
$$

and, recalling (12), we obtain

$$
|\xi|<\frac{6 U}{M h\left(\tilde{x}\left(\delta_{\tau}\right)|\nabla \eta(x)|\right)} .
$$

From Definition 1 we have

$$
\left\langle\frac{\nabla_{\xi} L}{L}\left(x, u, \xi_{\varepsilon}\right),-\eta \xi-u \nabla \eta\right\rangle \leq(\eta|\xi|+U|\nabla \eta|) K_{0} K_{2} \mathcal{L}^{\prime}\left(\left|\xi_{\varepsilon}\right|\right)
$$

noticing that

$$
\left|\xi_{\varepsilon}\right| \leq \frac{6 U}{\operatorname{Mh}\left(\tilde{x}\left(\delta_{\tau}\right)|\nabla \eta|\right)}+\varepsilon|\nabla \eta| U
$$

and that $\mathcal{L}^{\prime}$ is nondecreasing, from (10), (12), (17), (19), and (20) we obtain

$$
\begin{aligned}
& \ell_{\varepsilon}(x, u, \xi)-\log L(x, u, \xi) \\
& \quad \leq \varepsilon K U+\varepsilon(\eta|\xi|+U|\nabla \eta|) K_{0} K_{2} \mathcal{L}^{\prime}\left(\left|\xi_{\varepsilon}\right|\right) \\
& \quad \leq \varepsilon K U+\varepsilon|\nabla \eta| K_{0} K_{2} \mathcal{L}^{\prime}\left(\frac{6 U}{M \cdot h\left(\tilde{x}\left(\delta_{\tau}\right)|\nabla \eta|\right)}+\varepsilon|\nabla \eta| U\right)\left(\frac{6 U}{M}+U\right) .
\end{aligned}
$$

By (11), we have that $\tilde{x}\left(\delta_{\tau}\right)|\nabla \eta| \leq 1$; there exists $\sigma$ such that for $t<\sigma$ we have

$$
h(1) \leq \frac{1}{M U t}
$$

so that for $|\nabla \eta|<\sigma, h\left(\tilde{x}\left(\delta_{\tau}\right)|\nabla \eta|\right) \leq h(1) \leq \frac{1}{M U|\nabla \eta|}$. Then,

$$
\frac{6}{M \cdot h\left(\tilde{x}\left(\delta_{\tau}\right)|\nabla \eta|\right)}+\varepsilon|\nabla \eta| U \leq \frac{7}{M \cdot h\left(\tilde{x}\left(\delta_{\tau}\right)|\nabla \eta|\right)} .
$$


Hence, for those $x$ such that $|\nabla \eta(x)|<\sigma$, recalling (13), we obtain

$$
\begin{aligned}
|\nabla \eta| L^{\prime}\left(\frac{6 U}{M \cdot h\left(\tilde{x}\left(\delta_{\tau}\right)|\nabla \eta|\right)}+\varepsilon|\nabla \eta| U\right) & \leq|\nabla \eta| \mathcal{L}^{\prime}\left(\frac{7 U}{M \cdot h\left(\tilde{x}\left(\delta_{\tau}\right)|\nabla \eta|\right)}\right) \\
& =|\nabla \eta| \mathcal{L}^{\prime}\left(\partial\left(\mathcal{L}^{*}\right)\left(\frac{1}{\tilde{x}\left(\delta_{\tau}\right)|\nabla \eta|}\right)\right)=\frac{1}{\tilde{x}\left(\delta_{\tau}\right)},
\end{aligned}
$$

a constant independent on $\varepsilon$, thus proving the result in this case.

It is left to consider those $x$ such that $|\nabla \eta(x)| \geq \sigma$ : in this case, from (18), we have $|\xi| \leq \frac{6 U}{M \cdot h\left(\tilde{x}\left(\delta_{\tau}\right) \sigma\right)}$, and, from (10), the result follows from the boundedness of $|\nabla \eta|$.

3. The validity of the Euler-Lagrange equation. The higher integrability property for a minimizer $\tilde{u}$ is independent on the validity of the Euler-Lagrange equation. In the next theorem we wish to use this result in order to establish the validity of the Euler-Lagrange equation for a class of problems including Lagrangians having growth faster than exponential.

TheOrem 3. Let $L(x, u, \xi)$ satisfy Assumption A and assume that there exist a comparison function $\Lambda$ and a constant $c>0$ such that for $t \geq 1$ either

(i) $\frac{d}{d t} \mathcal{L}(t) \leq c$ or

(ii) $\frac{d}{d t} \mathcal{L}(t) \leq c(1+\log t), \mathcal{L}(\cdot)$ is convex, and $\operatorname{Dom}\left(\mathcal{L}^{*}\right)$ is open, where $\mathcal{L}(\cdot)=\log \Lambda(\cdot)$. Then, a locally bounded solution $\tilde{u}$ to the problem of minimizing

$$
\int_{\Omega} L(x, u(x), \nabla u(x)) \mathrm{d} x \text { for } \quad u \in u_{0}+W_{0}^{1,1}(\Omega)
$$

satisfies the Euler-Lagrange equation.

Lagrangians of exponential growth satisfy (i); the map $\Lambda(t)=t^{t}$ is not of exponential growth but satisfies (ii): in this case, $\operatorname{Dom}\left(\mathcal{L}^{*}\right)=\Re$.

In order to prove Theorem 3 , we shall need the following lemma.

Lemma 2. Let $L: \Re \rightarrow \Re$ be convex and $C^{1}$ and such that $\operatorname{Dom}\left(\partial L^{*}\right)$ is open; let $\delta^{*}$ be any selection from $\partial L^{*}$. Then, there exists a sequence of convex $C^{2}$ functions $L_{m}$ such that

(i) $\operatorname{Dom}\left(L_{m}^{\prime}\right) \supset[-m+1, m-1] ; \forall x \in \operatorname{Dom}\left(L_{m}^{\prime}\right)$, we have $\left|L_{m}^{\prime}(x)-L^{\prime}(x)\right|<\frac{1}{m}$;

(ii) $L_{m}^{*} \in C^{1}\left(\operatorname{Dom}\left(\partial L^{*}\right)\right)$; for every $[a, b] \subset \operatorname{Dom}\left(\partial L^{*}\right)$ there exists a subsequence $m(j)$ such that $\left(L_{m(j)}^{*}\right)^{\prime} \rightarrow \delta^{*}$ pointwise a.e. on $[a, b]$.

Proof. Ad (i). By assumption, $L^{\prime}$ is a single-valued, continuous, nondecreasing function; hence, its inverse, $\partial L^{*}$, is strictly increasing, possibly multivalued, and defined on the image of $L^{\prime}$. The selection $\delta^{*}$ (discontinuous at most on a set of measure zero) is strictly increasing and bounded on sets compactly contained in its domain. Consider the interval $[-n, n]$. The interval $\left[L^{\prime}(-n), L^{\prime}(n)\right]$ is a compact subset of the open set $\operatorname{Dom}\left(\partial L^{*}\right)$. There exists a subsequence $n(m)$ such that both $L^{\prime}(-n(m+1))<L^{\prime}(-n(m))$ and $L^{\prime}(n(m+1))>L^{\prime}(n(m))$; there exists $N(n(m))$, with $N(n(m)) \geq n(m)$ and $\frac{1}{N(n(m))} \leq \frac{1}{4} \min \left\{L^{\prime}(-n(m))-L^{\prime}(-n(m+1)), L^{\prime}(n(m+\right.$ $\left.1))-L^{\prime}(n(m))\right\}$, such that $\left[L^{\prime}(-n(m))-\frac{1}{N(n(m))}, L^{\prime}(n(m))+\frac{1}{N(n(m))}\right] \subset \operatorname{Dom}\left(\partial L^{*}\right)$, so that the map

$$
\left(L_{m}^{*}\right)^{\prime}=\rho_{N(n(m))} * \delta^{*},
$$

where $\rho_{N(n(m))}$ is a standard mollifier having support in $\left[-\frac{1}{N(n(m))}, \frac{1}{N(n(m))}\right]$, is well defined on $\left[L^{\prime}(-n(m)), L^{\prime}(n(m))\right]$ as a strictly increasing function. Its image is the 
interval $I(n(m))=\left[\left(L_{m}^{*}\right)^{\prime}\left(L^{\prime}(-n(m)),\left(L_{m}^{*}\right)^{\prime}\left(L^{\prime}(n(m))\right)\right]\right.$. We claim that $I(n(m+1)) \supset$ $[-n(m), n(m)] \supset[-m, m]$. In fact, consider $\bar{p}=\frac{1}{2}\left(L^{\prime}(-n(m+1))+L^{\prime}(-n(m))\right)$ : for every $p$ such that $|p-\bar{p}| \leq \frac{1}{N(n(m))}$, we have $\delta^{*}(p)<\delta^{*}\left(L^{\prime}(-n(m))\right)=-n(m)$ so that $\rho_{N(n(m+1))} * \delta^{*}(\bar{p})<-n(m)$, and analogously for $\frac{1}{2}\left(L^{\prime}(n(m+1))+L^{\prime}(n(m))\right)$.

The map $\left(L_{m}^{*}\right)^{\prime}$ is a $C^{1}$ and strictly increasing, hence invertible, function: on the interval $I(n(m))$ we set $L_{m}^{\prime}=\left(\left(L_{m}^{*}\right)^{\prime}\right)^{-1}$. Fix arbitrarily $m$ and $\bar{x} \in I(n(m))$. Set $\bar{y}_{m}=L_{m}^{\prime}(\bar{x})$ so that

$$
\bar{x}=\left(\rho_{N(n(m))} * \delta^{*}\right)\left(\bar{y}_{m}\right)=\int_{\left[\bar{y}_{m}-\frac{1}{N(n(m))}, \bar{y}_{m}+\frac{1}{N(n(m))}\right]} \rho_{N(n(m))}\left(\bar{y}_{m}-y\right) \delta^{*}(y) d y .
$$

We notice that $\bar{x} \in \operatorname{co}\left\{\delta^{*}(y):\left|y-\bar{y}_{m}\right| \leq \frac{1}{N(n(m))}\right\}$. In fact, otherwise, there exists $\alpha$ such that for every $y \in\left\{\left|y-\bar{y}_{m}\right| \leq \frac{1}{N(n(m))}\right\}$ we have $\alpha \bar{x}>\alpha \delta^{*}(y)$; then,

$$
\alpha \bar{x}=\int \alpha \bar{x} \cdot \rho_{N(n(m))}\left(\bar{y}_{m}-y\right)>\alpha \int \delta^{*}(y) \cdot \rho_{N(n(m))}\left(\bar{y}_{m}-y\right)=\alpha \bar{x} .
$$

Hence, there are $y_{1}$ and $y_{2}$ such that $\left|y_{i}-\bar{y}_{m}\right| \leq \frac{1}{N(n(m))}$ and $\delta^{*}\left(y_{1}\right) \leq \bar{x} \leq \delta^{*}\left(y_{2}\right)$. By the monotonicity of $L^{\prime}$, the last inequality can be written as $y_{1} \leq L^{\prime}(\bar{x}) \leq y_{2}$ so that

$$
\bar{y}_{m}-\frac{1}{N(n(m))} \leq y_{1} \leq L^{\prime}(\bar{x}) \leq y_{2} \leq \bar{y}_{m}+\frac{1}{N(n(m))}
$$

We have obtained

$$
\left|L^{\prime}(\bar{x})-L_{m}^{\prime}(\bar{x})\right| \leq \frac{1}{n(m)} \leq \frac{1}{m} .
$$

Ad (ii). Fix arbitrarily $[a, b]$. We have that $\left(L_{m}^{*}\right)^{\prime} \rightarrow \delta^{*}$ in $L^{1}([a, b])$. Hence, there exists a sequence $\left(L_{m(j)}^{*}\right)^{\prime}$ converging to $\delta^{*}$ pointwise a.e. on $[a, b]$.

The condition that $\operatorname{Dom}\left(\partial L^{*}\right)$ be open is not satisfied by a map like $L(t)=|t|$; it is satisfied by the minimal area functional $L(t)=\sqrt{1+|t|^{2}}$ and a fortiori by any $L$ of superlinear growth.

Proof of Theorem 3. Fix $0<h_{0}<\frac{1}{c}$; we claim that both in case (i) and in case (ii), there exists $K$ such that $0<h<h_{0}$ implies

$$
\Lambda^{\prime}(t+h) \leq K\left[1+\Lambda(t)+t \Lambda^{\prime}(t)\right] .
$$

Set $\Lambda^{*}=\sup \left\{\Lambda^{\prime}(s): 0 \leq s \leq 1+h_{0}\right\}$. In case (i), we have $\Lambda^{\prime}(t+h) \leq \Lambda^{*}$ for $t \leq 1$ while for $t>1, \Lambda(t+h) \leq c \Lambda(t) e^{c h}$, and we infer that (22) holds. In case (ii), again $\Lambda^{\prime}(t+h) \leq \Lambda^{*}$ for $t \leq 1$, while for $t>1$ we have

$$
\begin{aligned}
\Lambda^{\prime}(t+h) & =L^{\prime}(t+h) \frac{\Lambda(t+h)}{\Lambda(t)} \Lambda(t)=\mathcal{L}^{\prime}(t+h) \cdot \exp \left(\int_{t}^{t+h} \mathcal{L}^{\prime}(s) \mathrm{d} s\right) \cdot \Lambda(t) \\
& \leq c\left[1+\log \left(t+h_{0}\right)\right] \cdot \exp \left[c \int_{t}^{t+h_{0}}(1+\log s) \mathrm{d} s\right] \cdot \Lambda(t) \\
& \leq c\left(1+\log t+\frac{h_{0}}{t}\right) \cdot\left(t+h_{0}\right)^{c h_{0}} \cdot \exp \left[c t\left(\log \left(t+h_{0}\right)-\log t\right)\right] \Lambda(t) \\
& \leq c_{1}(1+\log t) \cdot t^{c h_{0}} e^{c h_{0}} \Lambda(t) \leq c_{2} \cdot t \Lambda(t) .
\end{aligned}
$$


By assumption, $\log (\Lambda)$ is convex for $t>1$ so that there exists $c_{3}$ such that $\frac{\Lambda^{\prime}(t)}{\Lambda(t)} \geq c_{3}$, hence $\Lambda^{\prime}(t+h) \leq c_{2} c_{3} \cdot t \Lambda^{\prime}(t)$, and (22) is established.

Next, we claim that setting $t=|\nabla \tilde{u}(x)|$ in the right-hand side of (22), we obtain a function integrable on compact subsets of $\Omega$. By (i) of the comparison assumption, we have that $\Lambda(|\nabla \tilde{u}|) \in L_{\text {loc }}^{1}(\Omega)$. By (ii) of the comparison assumption, to show that $|\nabla \tilde{u}| \Lambda^{\prime}(|\nabla \tilde{u}|) \in L_{l o c}^{1}(\Omega)$ it is enough to show that Theorem 1 holds, i.e., that the assumptions of Theorem 2 are satisfied. The assumptions are obviously satisfied in case (i), so we consider case (ii). We have to prove that

$$
\int^{\infty} \frac{1}{z \partial\left(\mathcal{L}^{*}\right)(z)} \mathrm{d} z<\infty
$$

Since $\operatorname{Dom}\left(\partial \mathcal{L}^{*}\right)$ is open, Lemma 2 can be applied to $\mathcal{L}$. Consider the sequence $\left(\mathcal{L}_{m}\right)$; for any $\alpha, \beta(\alpha \geq 1)$, by the change of variables $z=\mathcal{L}_{m}^{\prime}(t)$, we have

$$
\int_{\mathcal{L}_{m}^{\prime}(\alpha)}^{\mathcal{L}_{m}^{\prime}(\beta)} \frac{d z}{z\left(\mathcal{L}_{m}^{*}\right)^{\prime}(z)} \mathrm{d} z=\int_{\alpha}^{\beta} \frac{\mathcal{L}_{m}^{\prime \prime}(t)}{t \cdot \mathcal{L}_{m}^{\prime}(t)} \mathrm{d} t=\left.\frac{\log \mathcal{L}_{m}^{\prime}(t)}{t}\right|_{\alpha} ^{\beta}+\int_{\alpha}^{\beta} \frac{\log \mathcal{L}_{m}^{\prime}(t)}{t^{2}} \mathrm{~d} t
$$

By assumption (ii),

$$
\left.\frac{\log \mathcal{L}^{\prime}(t)}{t}\right|_{\alpha} ^{\beta}+\int_{\alpha}^{\beta} \frac{\log \mathcal{L}^{\prime}(t)}{t^{2}} \mathrm{~d} t \leq\left.\frac{\log (c(1+\log t))}{t}\right|_{\alpha} ^{\beta}+\int_{\alpha}^{\beta} \frac{\log (c(1+\log t))}{t^{2}} d t,
$$

and there exists $H$ such that for every $\alpha \geq 1$ and for every $\beta$, the right-hand side is bounded by $H$. Whenever $m-1 \geq \beta$, $\operatorname{Dom}\left(\mathcal{L}_{m}^{\prime}\right) \supset[\alpha, \beta]$ and $\left|\mathcal{L}^{\prime}-\mathcal{L}_{m}^{\prime}\right| \leq \frac{1}{m}$, so that the right-hand side of (24) is bounded by $H+2$ (independent of $\alpha, \beta$, and $m$ ). Consider the subsequence $m(j)$ provided by (ii) of Lemma 2. Fix any $a, b \in R$; let $\alpha$, $\beta$ such that for $j$ sufficiently large, $[a, b] \subset\left[\mathcal{L}_{m(j)}^{\prime}(\alpha), \mathcal{L}_{m(j)}^{\prime}(\beta)\right]$. By (ii) of Lemma 2 and by Fatou's lemma,

$$
\int_{a}^{b} \frac{1}{z \partial\left(\mathcal{L}^{*}\right)(z)} \mathrm{d} z=\int_{a}^{b} \frac{1}{z \delta^{*}(z)} \mathrm{d} z \leq \liminf \int_{a}^{b} \frac{d z}{z\left(\mathcal{L}_{m(j)}^{*}\right)^{\prime}(z)} \mathrm{d} z \leq H+2,
$$

so that (8) is satisfied and the integrability claim holds.

To establish the validity of the Euler-Lagrange equation, fix $\eta \in C_{c}^{1}(\Omega)$ (we assume $\sup \eta \leq 1)$ and set $\tilde{h}=\sup |\nabla \eta|$ and $S=\operatorname{spt}(\eta)$. Since $\tilde{u}$ is a solution, we have

$$
\int_{S} \frac{L(x, \tilde{u}(x)+\varepsilon \eta(x), \nabla \tilde{u}(x)+\varepsilon \nabla \eta(x))-L(x, \tilde{u}(x), \nabla \tilde{u}(x))}{\varepsilon} \mathrm{d} x \geq 0 ;
$$

the integrand converges pointwise to

$$
\left\langle\nabla_{\xi} L(x, \tilde{u}, \nabla \tilde{u}), \nabla \eta\right\rangle+\frac{\partial L}{\partial u}(x, \tilde{u}, \nabla \tilde{u}) \cdot \eta
$$

and we wish to dominate the integrand by an integrable function. We have

$$
\begin{aligned}
\left|\frac{L(x, \tilde{u}+\varepsilon \eta, \nabla \tilde{u}+\varepsilon \nabla \eta)-L(x, \tilde{u}, \nabla \tilde{u})}{\varepsilon}\right| \\
\leq\left|\frac{L(x, \tilde{u}+\varepsilon \eta, \nabla \tilde{u})-L(x, \tilde{u}, \nabla \tilde{u})}{\varepsilon}\right| \\
\quad+\left|\frac{L(x, \tilde{u}+\varepsilon \eta, \nabla \tilde{u}+\varepsilon \nabla \eta)-L(x, \tilde{u}+\varepsilon \eta, \nabla \tilde{u})}{\varepsilon}\right| \\
\leq\left|\frac{\partial L}{\partial u}(x, \tilde{u}+\bar{s} \varepsilon \eta, \nabla \tilde{u}) \cdot \eta\right|+\left|\left\langle\nabla_{\xi} L(x, \tilde{u}+\varepsilon \eta, \nabla \tilde{u}+\bar{t} \varepsilon \nabla \eta), \nabla \eta\right\rangle\right| .
\end{aligned}
$$


By Assumption $\mathrm{A}(\mathrm{i})$, the first term is bounded by $K L(x, \tilde{u}, \nabla \tilde{u}) \cdot e^{K} \cdot|\eta|$, an integrable function. Set $E=\{x:|\nabla \tilde{u}(x)| \geq 1+\tilde{h}\}$ and write the second term as

$$
\left|\left\langle\nabla_{\xi} L(x, \tilde{u}+\varepsilon \eta, \nabla \tilde{u}+\bar{t} \varepsilon \nabla \eta), \nabla \eta\right\rangle\right| \chi_{S \backslash E}+\left|\left\langle\nabla_{\xi} L(x, \tilde{u}+\varepsilon \eta, \nabla \tilde{u}+\bar{t} \varepsilon \nabla \eta), \nabla \eta\right\rangle\right| \chi_{E} .
$$

Set $U=\sup \{|\tilde{u}(x)|\}+1$. On $S \backslash E,|\nabla \tilde{u}+\varepsilon \nabla \eta| \leq 1+2 \tilde{h}=R$, and hence by Assumption $\mathrm{A}(\mathrm{ii})$, the first term is bounded by an integrable function. On $E$, we have $|\nabla \tilde{u}+\varepsilon \nabla \eta| \geq 1$; hence by (ii) of the comparison assumption and (22), whenever $\varepsilon \tilde{h}<h_{0}$,

$$
\begin{aligned}
\left|\left\langle\nabla_{\xi} L(x, \tilde{u}+\varepsilon \eta, \nabla \tilde{u}+\bar{t} \varepsilon \nabla \eta), \nabla \eta\right\rangle\right| & \leq K_{2} \Lambda^{\prime}(|\nabla \tilde{u}(x)+\bar{t} \varepsilon \nabla \eta|) \\
& \leq K_{2} K\left[1+\Lambda(|\nabla \tilde{u}(x)|)+|\nabla \tilde{u}(x)| \Lambda^{\prime}(|\nabla \tilde{u}(x)|)\right] .
\end{aligned}
$$

The last term is integrable, by our previous claim, and is independent of $\varepsilon$, so that we can pass to the limit under the integral sign. Finally, considering also $-\eta$, we obtain that

$$
\int_{\Omega}\left[\left\langle\nabla_{\xi} L(x, \tilde{u}(x), \nabla \tilde{u}(x)), \nabla \eta(x)\right\rangle+L_{u}(x, \tilde{u}(x), \nabla \tilde{u}(x)) \eta(x)\right] \mathrm{dx}=0
$$

for every admissible variation $\eta$.

Acknowledgment. This paper was improved by the sharp comments of an anonymous referee.

\section{REFERENCES}

[1] G. Bonfanti and M. Mazzola, On the validity of the Euler-Lagrange equation in a nonlinear case, Nonlinear Anal., 73 (2010), pp. 266-269.

[2] A. Cellina and M. Mazzola, Higher integrability for solutions to variational problems with fast growth, J. Convex Anal., 18 (2011), pp. 173-180.

[3] A. Cellina and M. Mazzola, Higher differentiability of solutions to variational problems, Calc. Var. Partial Differential Equations, to appear.

[4] F. H. Clarke, Optimization and Nonsmooth Analysis, 2nd ed., Classics Appl. Math. 5, SIAM, Philadelphia, 1990.

[5] M. Degiovanni and M. Marzocchi, On the Euler-Lagrange equation for functionals of the calculus of variations without upper growth conditions, SIAM J. Control Optim., 48 (2009), pp. $2857-2870$.

[6] G. M. Lieberman, On the regularity of the minimizer of a functional with exponential growth, Comment. Math. Univ. Carolin., 33 (1992), pp. 45-49.

[7] H. NAito, On a local Hölder continuity for a minimizer of the exponential energy functional, Nagoya Math. J., 129 (1993), pp. 97-113.

[8] R. T. Rockafellar, Convex Analysis, Princeton University Press, Princeton, NJ, 1972. 